\title{
Clozapine-induced acute gastrointestinal necrosis: a case report
}

\author{
Mark T. Osterman', Caitlin Foley ${ }^{1}$ and Isaac Matthias ${ }^{2^{*}}$ (I)
}

\begin{abstract}
Background: Clozapine is known to cause fecal impaction and ileus with resultant colonic necrosis due to compression of colonic mucosa. There are rare reports of clozapine causing necrosis of other portions of the gastrointestinal tract unrelated to constipation. We describe a case of acute necrosis of the upper gastrointestinal tract and small bowel to due to clozapine and quetiapine.

Case presentation: A 66-year-old white man with a past medical history of schizophrenia, maintained on clozapine and quetiapine, presented with hypoxic respiratory failure caused by aspiration of feculent emesis due to impacted stool throughout his colon. His constipation resolved with discontinuation of clozapine and quetiapine, and his clinical condition improved. These medicines were restarted after 2 weeks, resulting in acute gastrointestinal necrosis from the mid esophagus through his entire small bowel. He died due to septic shock with Gram-negative rod bacteremia.
\end{abstract}

Conclusions: Clozapine may cause acute gastrointestinal necrosis.

Keywords: Clozapine, Quetiapine, Antipsychotic, Acute gastrointestinal necrosis, Acute bowel necrosis, Acute esophageal necrosis

\section{Background}

Constipation is a common side effect of antipsychotic medicines, and is attributed to their anticholinergic properties [1]. Constipation due to antipsychotics can progress to severe pathology such as ileus, fecal impaction, aspiration of feculent emesis, and colonic ischemia due to compression of colonic mucosa [2]. Life-threatening complications of constipation due to antipsychotic medication occur most frequently with clozapine, and are estimated to occur in $0.3 \%$ of patients who receive this medicine [3, 4]. It is not certain whether clozapine can cause ischemia in portions of the gastrointestinal tract other than the colon, unrelated to constipation. We present a case of a patient who developed acute necrosis of the esophagus, stomach, and small bowel shortly after clozapine re-initiation.

\footnotetext{
* Correspondence: Isaac.Matthias@uphs.upenn.edu

${ }^{2}$ Section of Hospital Medicine, Perelman School of Medicine, University of Pennsylvania, Philadelphia, Pennsylvania, USA

Full list of author information is available at the end of the article
}

\section{Case presentation}

A 66-year-old white man with a history of schizophrenia presented to our emergency department with acute altered mental status and feculent emesis. His abdomen was distended but not tender. He was found to be in severe respiratory distress requiring endotracheal intubation, and shock requiring vasopressor infusion. Computed tomography (CT) imaging revealed acute respiratory distress syndrome (ARDS) due to aspiration as well as impacted stool throughout his colon. Home antipsychotic medicines, clozapine and quetiapine, were held, and after manual disimpaction and enemas he had copious bowel movements and resolution of abdominal distension. His shock and ARDS gradually improved. Vasopressors were discontinued on day 3 , and he remained normotensive. In-patient medicines were carbamazepine, lansoprazole, phenytoin, polyethylene glycol, senna, and subcutaneous heparin prophylaxis. Clozapine $100 \mathrm{mg}$ every 12 hours was restarted on day 11 , and a single dose of quetiapine $400 \mathrm{mg}$ was given on day 16 , both at home doses. Six hours after quetiapine was restarted, he was noted to have recurrence of abdominal distension and new onset of copious foul-smelling hematemesis. 


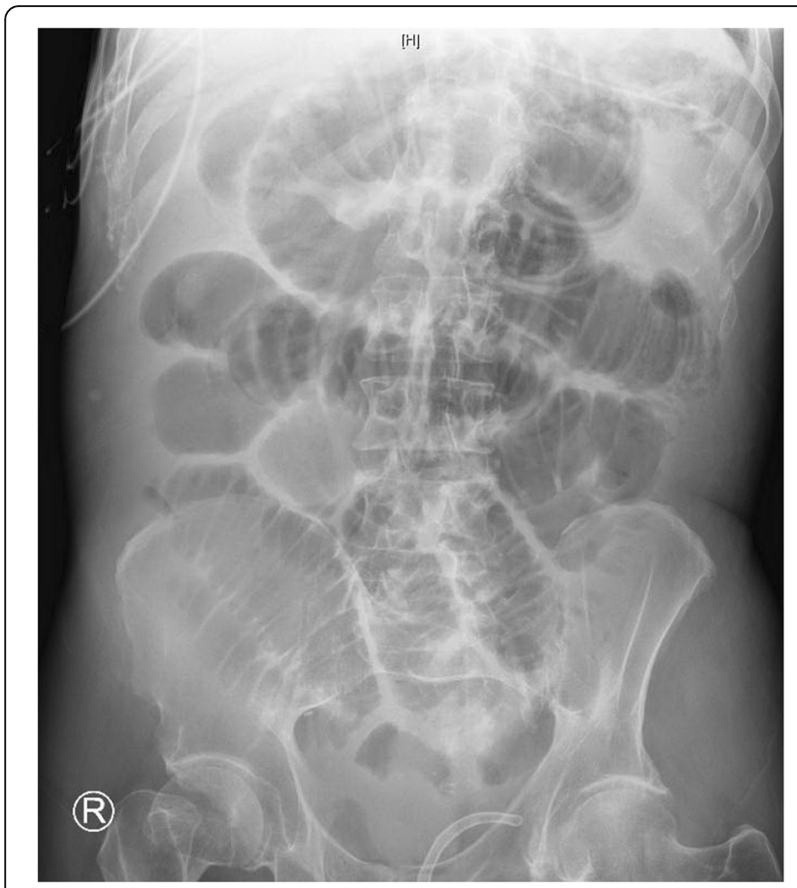

Fig. 1 Abdominal X-ray showing diffuse small bowel dilation
An abdominal X-ray showed diffuse small bowel dilation (Fig. 1). An urgent esophagogastroduodenoscopy (EGD) was performed, showing transition from normal mucosa in the proximal esophagus to severe diffuse ulceration beginning in the mid esophagus (Fig. 2a), with continued severe ulceration throughout the distal esophagus (Fig. 2b), stomach (Fig. 2c), and duodenum (Fig. 2d). He developed progressive septic shock, and blood cultures ultimately grew Escherichia coli and Klebsiella oxytoca. Surgical consultation was obtained, but no surgical intervention was possible given the widespread gastrointestinal involvement and his moribund state. He died within 24 hours of developing symptoms. An autopsy was declined.

\section{Discussion}

Our patient's initial presentation with aspiration of feculent emesis due to impacted stool throughout the colon is within the known spectrum of life-threatening clozapineinduced colonic hypomotility. Fecal impaction resolved with discontinuation of clozapine and quetiapine.

Acute gastrointestinal necrosis developed 5 days after restarting clozapine and 6 hours after restarting quetiapine. EGD and abdominal X-ray were consistent with necrosis from the mid esophagus through the entire small bowel. His new onset foul smelling hematemesis, abdominal distension, and polymicrobial Gram-negative

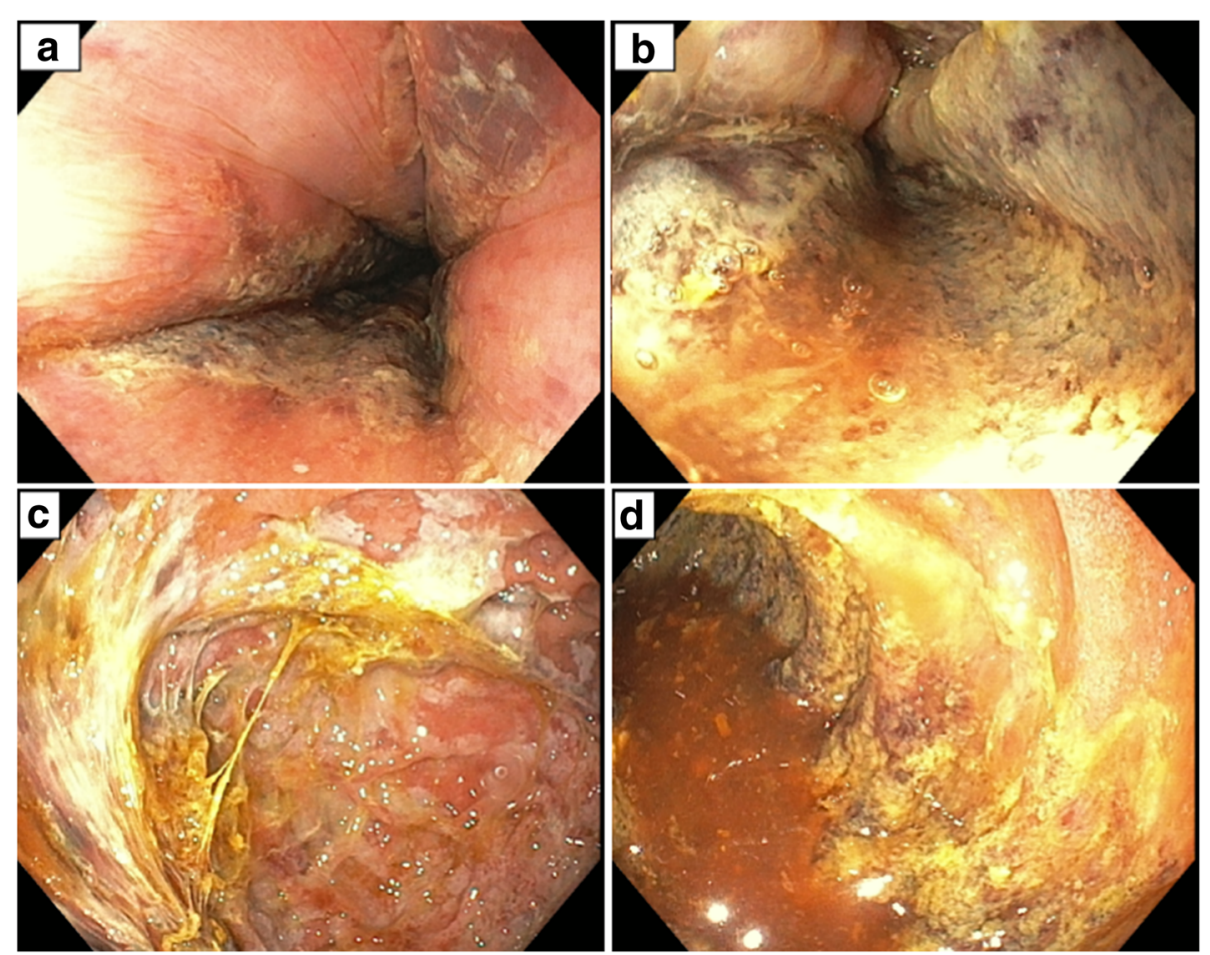

Fig. 2 Esophagogastroduodenoscopy images showing severe diffuse ulceration of the upper gastrointestinal tract. a Transition from normal to ulcerated mucosa in the mid esophagus, b distal esophagus, c stomach, and $\mathbf{d}$ duodenum 
rod bacteremia clinically matched imaging findings, and he died within 24 hours of developing symptoms. Given the temporal association with his clinical deterioration, we believe his acute gastrointestinal necrosis was caused by re-initiating clozapine and quetiapine.

Other causes of gastrointestinal necrosis were considered. It is unlikely that proximal splanchnic arterial occlusion from embolic or other source occurred, as necrosis was present in multiple vascular territories. Our patient had remained off vasopressors with normal blood pressure for 13 days, making a shock state an unlikely cause. His other medications did not include vasoactive drugs known to cause mesenteric ischemia such as alpha agonists, digoxin, or ergotamines [5].

There are few reports of clozapine causing acute necrosis of portions of the gastrointestinal tract other than the colon. Yu et al. in 2013 reported a case of a 47-yearold man who developed acute necrosis of small bowel and colon 6 days after initiating clozapine [6]. Pautola and Hakala in 2016 reported a case of a 65-year-old man who developed acute esophageal necrosis 6 hours after receiving a single dose of clozapine and olanzapine due to medication error [7]. As in our patient, these cases occurred shortly after initiating clozapine, involved portions of the gastrointestinal tract outside the colon, and were unrelated to constipation.

A possible mechanism of clozapine-induced acute gastrointestinal necrosis is antiserotonergic effect. Clozapine is the only antipsychotic medicine known to be a competitive inhibitor of the 5-hydroxytryptamine ${ }_{3}$ (5$\mathrm{HT}_{3}$ ) serotonin receptor [8]. Alosetron, a potent $5-\mathrm{HT}_{3}$ inhibiting drug used to treat irritable bowel syndrome with diarrhea, was withdrawn from the market due to causing ischemic colitis unrelated to constipation, and was subsequently reintroduced with a black box warning $[9,10]$.

\section{Conclusions}

Clozapine is known to cause fecal impaction and ileus, compressing the colonic mucosa and leading to colonic necrosis. Two recent cases have described clozapineinduced acute necrosis of the small bowel and upper gastrointestinal tract, without constipation. We present a third such case. Further monitoring is needed to confirm this suspected life-threatening side effect of clozapine.

\footnotetext{
Abbreviations

5-HT3: 5-Hydroxytryptamine3; ARDS: Acute respiratory distress syndrome; $\mathrm{CT}$ : Computed tomography; EGD: Esophagogastroduodenoscopy
}

Availability of data and materials

Not applicable.

\begin{abstract}
Authors' contributions
IM was the primary care provider during the in-patient hospitalization. CF and $\mathrm{MO}$ provided gastroenterology consultation and performed upper endoscopy. IM wrote the initial draft of the manuscript. All authors reviewed and contributed to the final version of this case report. All authors read and approved the final manuscript.
\end{abstract}

Ethics approval and consent to participate

Not applicable.

\section{Consent for publication}

Written informed consent was obtained from the patient's next of kin for publication of this case report and any accompanying images. A copy of the written consent is available for review by the Editor-in-Chief of this journal.

\section{Competing interests}

The authors declare that they have no competing interests.

\section{Publisher's Note}

Springer Nature remains neutral with regard to jurisdictional claims in published maps and institutional affiliations.

\section{Author details}

${ }^{1}$ Division of Gastroenterology, Perelman School of Medicine, University of Pennsylvania, Philadelphia, Pennsylvania, USA. ${ }^{2}$ Section of Hospital Medicine, Perelman School of Medicine, University of Pennsylvania, Philadelphia, Pennsylvania, USA.

Received: 3 August 2017 Accepted: 8 September 2017

Published online: 23 September 2017

References

1. De Hert M, Hudyana H, Dockx L, et al. Second-generation antipsychotics and constipation: a review of the literature. Eur Psychiatry. 2011;26(1):34-44 [PubMed].

2. De Hert M, Dockx L, Bernagie C, et al. Prevalence and severity of antipsychotic related constipation in patients with schizophrenia: a retrospective descriptive study. BMC Gastroenterol. 2011;11:17 [PubMed].

3. Nielsen J, Meyer J. Risk factors for ileus in patients with schizophrenia. Schizophr Bull. 2012;38(3):592-8 [PubMed].

4. Palmer S, McLean R, Ellis P, Harrison-Woolrych M. Life-threatening clozapineinduced gastrointestinal hypomotility: an analysis of 102 cases. J Clin Psychiatry. 2008;69(5):759-68 [PubMed].

5. Bobadilla J. Mesenteric ischemia. Surg Clin North Am. 2013;93(4):925-40. ix [PubMed].

6. Yu S, Chen H, Lee S. Rapid development of fatal bowel infarction within 1 week after clozapine treatment: a case report. Gen Hosp Psychiatry. 2013; 35(6):679. e5-6. [PubMed].

7. Pautola L, Hakala T. Medication-induced acute esophageal necrosis: a case report. J Med Case Rep. 2016;10(1):267 [PubMed].

8. Rammes G, Eisensamer B, Ferrari U, et al. Antipsychotic drugs antagonize human serotonin type 3 receptor currents in a noncompetitive manner. Mol Psychiatry. 2004;9(9):846-58. 818 [PubMed].

9. Chang L, Tong K, Ameen V. Ischemic colitis and complications of constipation associated with the use of alosetron under a risk management plan: clinical characteristics, outcomes, and incidences. Am J Gastroenterol. 2010;105(4):866-75 [PubMed].

10. Friedel D, Thomas R, Fisher R. Ischemic colitis during treatment with alosetron. Gastroenterology. 2001;120(2):557-60 [PubMed]. 